\title{
Chromosome Synapsis During Gametogenesis of Humanized Mice Carrying a Point Mutation in the Syce1 Gene.
}

Hernández-López $\mathrm{D}^{1}$, Benavente $\mathrm{R}^{2}$, Geisinger $\mathrm{A}^{1,3}$, Trovero $\mathrm{F}^{1,4}$, Santiñaque $\mathrm{FF}^{5}$, Folle $\mathrm{GA}^{4,5}$, Rodríguez-Casuriaga $\mathbf{R}^{1,3^{*}}$

1. Department of Molecular Biology, Instituto de Investigaciones Biológicas Clemente Estable (IIBCE), 11,600 Montevideo, Uruguay.

2. Department of Cell and Developmental Biology, Biocenter, University of Würzburg, D97074 Würzburg, Germany.

${ }^{3}$ Biochemistry-Molecular Biology, Facultad de Ciencias, Universidad de la República (UdelaR), 11,400 Montevideo, Uruguay

4. Department of Genetics, IIBCE, 11,600 Montevideo, Uruguay.

5. Flow Cytometry and Cell Sorting Core, IIBCE, 11,600 Montevideo, Uruguay.

*Corresponding author: rrodriguez@iibce.edu.uy

More than $50 \%$ of cases of premature ovarian failure and non-obstructive azoospermia in humans are classified as idiopathic infertility (unknown cause). Meiotic defects may relate to at least part of these cases. Mutations in genes coding for synaptonemal complex (SC) components have been reported in humans, and hypothesized to be at the groundwork of the observed infertile phenotype. Two such mutations have been reported for the SC central element component - coding gene Sycel[1,2].

In order to investigate the actual consequences of one of these Sycel point mutations, we have used the CRISPR/Cas9 technology to generate a mouse model line with an equivalent genome alteration. We hereby present the characterization of the mutant mice phenotype, as contrasted to their wild type and heterozygous littermates. The studies performed included fertility tests, flow cytometry profiling of testicular cell suspensions, cytology analysis, and immunolocalizations on cryosections and spread cells, among others. Increased resolution of SC structure was achieved through an AIRYSCAN super-resolution module, coupled to a Zeiss 800 LSM inverted microscope.

Main representative results are as follows: a) CRISPR/Cas9 technology was successfully applied to generate a mouse model line with the desired mutation; $b$ ) the introduced point mutation proved to be the actual cause of the infertile phenotype, both in male and female mice homozygous for the change; c) the mechanisms that lead to infertility in these mutants are related to meiotic defects; d) fine analysis of spread chromosomes showed alterations in SC assembly and chromosome synapsis.

As far as we know, this is the first report on the employment of CRISPR/Cas technology to direct a specific change to a SC component, and generate a humanized mouse model line for its exhaustive study [3]. 


\section{References:}

[1] L. de Vries et al., J. Clin. Endocrinol. Metab. 99 (E2) (2014) p. 129.

[2] E. Maor-Sagie et al., J. Assist. Reprod. Genet. 32 (2015) p. 887.

[3] The authors acknowledge funding from Agencia Nacional de Investigación e Innovación (ANII, Uruguay) (project ANII-FCE-3-2016-1-126285 to R.R-C). Dr. Mónica Brauer is thanked for her invaluable help with embryonic ovary dissections. 